\title{
Fuzzy Rough Set based Attribute Reduction in Fuzzy Decision Tables
}

\author{
Cao Chinh Nghia \\ People's Police Academy, \\ Ha Noi, Viet Nam
}

\author{
Nguyen Long Giang \\ Institute of Information Technology, \\ VAST, Ha Noi, Vietnam
}

\begin{abstract}
Attributes reduction methods based approach traditional rough sets perform on the decision tables with discrete attribute value domain. In fact the data is usually the real values or symbols should be reduced attributes of traditional rough sets proved ineffective because of its failure to preserve the difference of data on original objects. This problem is solved with the attributes reduction methods based approach fuzzy rough set to overcome the limitations of the method according to previous rough set approach. This paper improves, analyzes and evaluates two methods of attribute reduction based on the degree of dependence between attributes and discernibility matrix of fuzzy rough set
\end{abstract}

\section{General Terms}

Data Mining, Rough Set Theory, Fuzzy Set, Fuzzy Rough Set

\section{Keywords}

Fuzzy rough set, fuzzy decision table, discernibility matrix, attributes reduction, reduct.

\section{INTRODUCTION}

Attributes reduction is important problems in data preprocessing steps aimed at reducing the dimensionality of data (number of attributes) in order to increase the efficiency of data mining algorithms and machine learning. Rough set theory proposed by Pawlak [5] is seen as effective tools to solve the problem of reduced attributes. Attributes reduction methods based approach traditional rough sets perform on the decision tables with discrete attribute value domain. In fact, the attribute value domain of decision table is often continuously for containing the real values (real-valued) or symbols (symbolic). To solve this problem, rough set theory using discrete methods of data before performing the attributes reduction methods. However, the degree of dependence of the discrete values is not considered. For example, two initial attribute values of temperature 39.5 and 39,6 are converted to the same value "high temperature" but does not show the temperature deviation is 0.1 . Thus, the discrete method does not preserve semantics of the original data. To solve this problem, D. Dubois and colleagues propose fuzzy rough set model [3] combines rough set theory [5] and fuzzy set theory [4]. Fuzzy set theory serves to preserve semantics of the data, while the rough set theory preserve not distinguish data. Similar to the traditional model of rough set, fuzzy rough set models using fuzzy similarity relation to approximate the fuzzy sets into sets of lower approximation and upper approximation [2]. So far, many works have studied this issue, focusing mainly on the construction of the lower approximation, upper approximation and attributes reduction [2], [3], [8], [9]. The results of attribute reduction using fuzzy rough set model is an extension of the traditional rough set, mainly focused on two directions is reduced attributes based on fuzzy partition and fuzzy similarity relations.
Based on approach attributes reduction methods of traditional rough set theory [1], [6], this paper improves, analyzes attributes reduction methods in decision table (system) using the degree of dependency between attributes in fuzzy rough set model, analyze advantages and disadvantages of the methods. In the next part, we present attributes reduction method based on discernibility matrix by fuzzy rough set approach and analyze advantages and disadvantages of the method. Two illustrative examples of methods used on the same set of data shows the feasibility of the method according to the fuzzy rough set approach in practice [2], [7], [10] compared to the corresponding method in the way to traditional rough set approach. The structure of paper as follows. Section 2 presents some basic concepts and attributes reduction methods used the degree of dependency between attributes in the model of fuzzy rough set, this is a typical method of research attributes reduction as assigned fuzzy partition. Section 3 presents some basic concepts in fuzzy rough set model and an attribute reduction method based on discernibility matrix, the typical method of research attributes reduction based on fuzzy similarity relations. Finally conclusions and development trend continued.

\section{ATTRIBUTE REDUCTION BASED ON THE DEPENDENCE BETWEEN ATTRIBUTES}

Model of fuzzy rough set is based on a combination of rough set theory and fuzzy set theory to approximate the fuzzy sets use fuzzy similarity relation [3], [8], [9]. A fuzzy similarity relation $\mathrm{S}$ on Universe $\mathrm{U}$ satisfying three properties: reflexive $\left(\mu_{S}(x, x)=1\right), \quad$ symmetry $\quad\left(\mu_{S}(x, y)=\mu_{S}(y, x) \quad\right.$ and transitivity $\left(\mu_{S}(x, z) \geq \mu_{S}(x, y) \wedge \mu_{S}(y, z)\right)$. Similarly in the traditional rough set theory based on fuzzy similarity relation, each set of attributes in $P \subseteq A$ defining a fuzzy partition as follows:

$$
U / P=\otimes\{a \in P: U / I N D(\{a\})\}
$$

Where

$$
A \otimes B=\{X \cap Y: \forall X \in A, \forall Y \in B, X \cap Y \neq \varnothing\}
$$

Each element in $U / P$ is a fuzzy equivalence class $[x]_{P}$ where $\mu_{[x]_{p}}(y)=\mu_{P}(x, y)$.

$$
\begin{aligned}
& \text { Example: Suppose that } P=\{a, b\}, \\
& U / I N D(\{a\})=\left\{N_{a}, Z_{a}\right\}, \quad U / I N D(\{b\})=\left\{N_{b}, Z_{b}\right\} \quad \text { Then } \\
& U / P=\left\{N_{a} \cap N_{b}, N_{a} \cap Z_{b}, Z_{a} \cap N_{b}, Z_{a} \cap Z_{b}\right\}
\end{aligned}
$$

The membership of the objects in equivalence classes are defined based on fuzzy rough set theory [2], [3]: 


$$
\mu_{F_{1} \cap \ldots \cap F_{n}}(x)=\min \left(\mu_{F_{1}}(x), \mu_{F_{2}}(x), \ldots, \mu_{F_{n}}(x)\right)
$$

Based on fuzzy equivalence classes, the concepts of lower approximation and upper approximation are expanded into fuzzy lower approximation and fuzzy upper approximation. With attribute sets $P \subseteq A$, the membership of the objects belonging to the fuzzy lower approximation and fuzzy upper approximation are defined [8], [9]:

$$
\begin{aligned}
& \mu_{\underline{\underline{X}}}(x)=\sup _{F \in U / P} \min \left(\mu_{F}(x), \inf _{y \in U} \max \left\{1-\mu_{F}(y), \mu_{X}(y)\right\}\right)(3) \\
& \mu_{\bar{P} X}(x)=\sup _{F \in U / P} \min \left(\mu_{F}(x), \sup _{y \in U} \min \left\{\mu_{F}(y), \mu_{X}(y)\right\}\right)
\end{aligned}
$$

Where inf $X, \sup X$ respectively is the lower right and upper right of the set $\mathrm{X}$. $\mathrm{F}$ is the fuzzy equivalence classes of fuzzy partition. $\langle\underline{P} X, \bar{P} X\rangle$ called a fuzzy rough set.

Decision table (system) is a special kind of information systems, which include a set of attributes A separates two subsets: the set of conditional attributes $\mathrm{C}$ and decision attributes D. Thus, decision table is an information system $D S=(U, C \cup D)$ with $C \cap D=\varnothing$. Decision table DS is called consistent if and only if $\operatorname{POS}_{C}(D)=U$, contrary DS is inconsistent [1].

Attributes reduction is the process of selecting the smallest subset of attributes conditions that preserve information classification decision table, called the collection of reduct (reduct). According to the traditional rough set approach, Pawlak [6] launched a collective set concepts based on positive region and constructive heuristic algorithm to find the best set of decision tables based on the evaluation criteria of importance attribute.

Definition 1. [6] Let $D S=(U, C \cup D)$ be the decision table and $R \subseteq C$. If

$$
\begin{aligned}
& \text { 1) } \operatorname{POS}_{R}(D)=\operatorname{POS}_{C}(D) \\
& \text { 2) } \forall r \in R, P O S_{R-\{r\}}(D) \neq P O S_{C}(D)
\end{aligned}
$$

then $\mathrm{R}$ is the collection of $\mathrm{C}$ base on positive region

Definition 2. [1] Let $D S=(U, C \cup D)$ be the decision table, $B \subset C$ and $b \in C-B$. The importance degree of attribute $b$ which respect to attribute set $B$ is defined by:

$$
S_{B} G_{B}(b)=\gamma_{B \cup\{b\}}(D)-\gamma_{B}(D)=\frac{\left|P_{B \cup S_{B \backslash b\}}(D) \mid}\right|-\left|P_{B}(D)\right|}{|U|}
$$

Suppose that $\left|\operatorname{POS}_{\varnothing}(D)\right|=0$. It is easy to see that $\left|P O S_{B \cup\{b\}}(D)\right| \geq\left|P O S_{B}(D)\right| \quad$ lead $\quad$ to $\quad S_{B}(b) \geq 0$. $S I G_{B}(b)$ is calculated by the amount of change in the dependence of $\mathrm{B}$ into $\mathrm{D}$ when adding attribute $\mathrm{b}$ to $\mathrm{B}$ and the greater $\operatorname{SIG}_{B}(b)$ is, the more amount of change is, as important attribute $b$ and vice versa. The importance degree of this attribute is the attribute selection criteria in heuristic algorithm to find the collection of decision table. Fuzzy decision table is the decision table which conditional attributes receive fuzzy values.

From Definition 1 combined with the definition of the degree of dependency between the attributes in the formula (5), attribute sets $R \subseteq C$ called collective reduction of $\mathrm{C}$ based on positive region if $\quad \gamma_{R}(D)=\gamma_{C}(D) \quad$ and $\forall r \in R, \gamma_{R-\{r\}}(D) \neq \gamma_{C}(D)$.

In traditional rough set theory, the concept of positive region is defined as the intersection of all lower approximation sets. With $P, Q \subseteq A$, the membership of objects in fuzzy positive region of fuzzy rough set model is defined [8], [9]

$$
\mu_{P O S_{p}(Q)}(x)=\sup _{X \in U / Q} \mu_{\underline{P} X}(x)
$$

Based on the concept of fuzzy positive region, the fuzzy membership perform the degree of dependence between attributes is defined [7]:

$$
\gamma_{P}^{\prime}(Q)=\frac{\left|\mu_{P O S_{P}(Q)}(x)\right|}{|U|}=\frac{\sum_{x \in U} \mu_{P O S_{P}(Q)}(x)}{|U|}
$$

The importance of attributes using fuzzy membership in the formula (7) is described as follows:

$$
S I G_{B}(b)=\gamma_{B \cup\{b\}}^{\prime}(D)-\gamma_{B}^{\prime}(D)
$$

Similar attributes reduction algorithm using the degree of dependence between the attributes in traditional rough set [1], attributes reduction algorithm in fuzzy decision table using membership in the formula (7) are described as follows:

Algorithm 1. Heuristic algorithm finds a minimal reduct set using membership functions.

Input: A decision table $D S=(U, C \cup D)$

Output: A minimal reduct $R$

1. $R \leftarrow \varnothing$;

2. $\gamma_{\varnothing}^{\prime}(D)=0$;

3. While $\gamma_{R}^{\prime}(D) \neq \gamma_{C}^{\prime}(D)$ do

4. Begin

5. For $c \in C-R$ calculate

$$
S I G_{R}(c)=\gamma_{R \cup\{c\}}^{\prime}(D)-\gamma_{R}^{\prime}(D) ;
$$

6. Select $c_{m} \in C-R$ so that

$$
S I G_{R}\left(c_{m}\right)=\underset{c \in C-R}{\operatorname{Max}}\{\operatorname{SIG}(c)\} ;
$$

7. $\quad R \leftarrow R \cup\left\{c_{m}\right\}$;

8. End;

9. Return $R$;

Example 1. Suppose that the decision table $D S=(U, C \cup\{d\})$ is described in table 1 . 
Table 1. Decision table describe in example 1

\begin{tabular}{|c|c|c|c|c|}
\hline Object & $\boldsymbol{a}$ & $\boldsymbol{b}$ & $\boldsymbol{c}$ & $\boldsymbol{d}$ \\
\hline 1 & -0.4 & -0.3 & -0.5 & No \\
\hline 2 & -0.4 & 0.2 & -0.1 & Yes \\
\hline 3 & -0.3 & -0.4 & -0.3 & No \\
\hline 4 & 0.3 & -0.3 & 0 & Yes \\
\hline 5 & 0.2 & -0.3 & 0 & Yes \\
\hline 6 & 0.2 & 0 & 0 & No \\
\hline
\end{tabular}

Assume that the value of the attribute $a, b$ and $c$ are represented by two fuzzy sets Fuzzy sets $\mathrm{N}$ and $\mathrm{Z}$. $\mathrm{N}$ fuzzy trapezoidal shaped square, is determined the membership function as follows:

1) $\mu_{N}(x)=0$ with $x<-1$

2) $\mu_{N}(x)=1$ with $-1 \leq x \leq-0.5$

3) $\mu_{N}(x)=\frac{-u}{0.5}$ with $-0.5<x<0$

4) $\mu_{N}(x)=0$ with $x \geq 0$

$\mathrm{Z}$ fuzzy triangular shape, the membership function is determined as follows:

1) $\mu_{z}(x)=0$ with $x<-0.5$

2) $\mu_{z}(x)=\frac{x+0.5}{0.5}$ with $-0.5 \leq x \leq 0$

3) $\mu_{z}(x)=\frac{0.5-x}{0.5}$ with $0<x \leq 0.5$

4) $\mu_{Z}(x)=0$ with $x>0.5$

Thus, the decision table in Example 1 combines with two fuzzy $\mathrm{N}$ and $\mathrm{Z}$ gives us the new decision table $D S^{\prime}=\left(U, C^{\prime} \cup\{d\}\right)$ with attributes $A=\{a\}, B=\{b\}, C=\{c\}, D=\{d\}$. The equivalence classes derived from attributes $A, B, C$, respectively: $U / A=\left\{N_{a}, Z_{a}\right\}, \quad U / B=\left\{N_{b}, Z_{b}\right\}, \quad U / C=\left\{N_{c}, Z_{c}\right\}$, $U / D=\{\{1,3,6\},\{2,4,5\}\}$; where $N_{a}, Z_{a}$ is two fuzzy sets defined on the attributes a; $N_{b}, Z_{b}$ is two fuzzy sets defined on the attributes $\mathrm{b} ; N_{c}, Z_{c}$ is two fuzzy sets defined on the attributes $\mathrm{c}$

Table 2. Decision table described Example 1

\begin{tabular}{|c|c|c|c|c|c|c|c|}
\hline \multirow{2}{*}{ Object } & \multicolumn{2}{|c|}{ A } & \multicolumn{2}{|c|}{ B } & \multicolumn{2}{|c|}{ C } & \multirow{2}{*}{ d } \\
\cline { 2 - 7 } & $\mathbf{N a}$ & $\mathbf{Z a}$ & $\mathbf{N b}$ & $\mathbf{Z b}$ & $\mathbf{N c}$ & $\mathbf{Z c}$ & \\
\cline { 2 - 7 } & $\boldsymbol{C}_{\mathbf{1}}$ & $\boldsymbol{C}_{\mathbf{2}}$ & $\boldsymbol{C}_{\mathbf{3}}$ & $\boldsymbol{C}_{\mathbf{4}}$ & $\boldsymbol{C}_{\mathbf{5}}$ & $\boldsymbol{C}_{\mathbf{6}}$ & \\
\hline 1 & 0.8 & 0.2 & 0.6 & 0.4 & 1 & 0 & No \\
\hline 2 & 0.8 & 0.2 & 0 & 0.6 & 0.2 & 0.8 & Yes \\
\hline
\end{tabular}

\begin{tabular}{|c|c|c|c|c|c|c|c|}
\hline 3 & 0.6 & 0.4 & 0.8 & 0.2 & 0.6 & 0.4 & No \\
\hline 4 & 0 & 0.4 & 0.6 & 0.4 & 0 & 1 & Yes \\
\hline 5 & 0 & 0.6 & 0.6 & 0.4 & 0 & 1 & Yes \\
\hline 6 & 0 & 0.6 & 0 & 1 & 0 & 1 & No \\
\hline
\end{tabular}

Apply steps of the algorithm 1 finds reductive set of decision table had provided, first steps we must calculate the lower approximation set for the attributes $\mathrm{A}, \mathrm{B}$ and $\mathrm{C}$. Considering the attributes A, with equivalent classes $X=\{1,3,6\}, \mu_{\underline{\underline{A}\{1,3,6\}}}\{x\}$ is calculated:

$\mu_{\underline{\underline{A}\{1,3,6\}}}(x)=\sup _{F \in U / A} \min \left(\mu_{F}(x), \inf _{y \in U} \max \left\{1-\mu_{F}(y), \mu_{\{1,3,6\}}(y)\right\}\right)$

Considering the fuzzy equivalence class $N_{a}$ on attribute A:

$$
\min \left(\mu_{N_{a}}(x), \inf _{y \in U} \max \left\{1-\mu_{N_{a}}(y), \mu_{\{1,3,6\}}(y)\right\}\right)
$$

Object 1 is calculated:

$$
\min (0.8, \inf \{1,0.2,1,0.4,1,1\})=0.2
$$

Similarly for $Z_{a}$

$$
\min (0.2, \inf \{1,0.8,1,0.6,0.4,1\})=0.2
$$

Thus $\mu_{\underline{A}\{1,3,6\}}(1)=0.2$. Calculate A-approximately the lower of $X=(1,3,6)$ for other objects in the same way we have: $\quad \mu_{\underline{A}\{1,3,6\}}(1)=0.2, \quad \mu_{\underline{A}\{1,3,6\}}(2)=0.2$, $\mu_{\underline{A}\{1,3,6\}}(3)=0.4, \quad \mu_{\underline{A}\{1,3,6\}}(4)=0.4 \quad \mu_{\underline{A}\{1,3,6\}}(5)=0.4$, $\mu_{\underline{A}\{1,3,6\}}(6)=0.4$.

Similarity with equivalence classes $X=\{2,4,5\}, \mu_{\underline{A}\{2,4,5\}}\{x\}$, fuzzy positive region for objects is calculated by the formula:

$$
\mu_{P O S_{A}(Q)}(x)=\sup _{X \in U / Q} \mu_{\underline{A} X}(x)
$$

We obtained: $\quad \mu_{P_{O S}(Q)}(1)=0.2, \quad \mu_{P_{A O S}(Q)}(2)=0.2$, $\mu_{P^{\prime} S_{A}(Q)}(3)=0.4, \quad \quad \mu_{P_{O S}(Q)}(4)=0.4$, $\mu_{P_{O S}(Q)}(5)=0.4, \mu_{P_{A O S}(Q)}(6)=0.4$. Since then, the membership of $\mathrm{Q}$ on $\mathrm{A}$ is calculated:

$$
\gamma_{A}^{\prime}(Q)=\frac{\sum_{x \in U} \mu_{P_{O S S_{A}(Q)}}(x)}{|U|}=2 / 6
$$

Similar to attribute B and C, we have: $\gamma_{B}^{\prime}(Q)=2.4 / 6$, $\gamma_{C}^{\prime}(Q)=1.6 / 6$. Attribute B has the largest membership functions included the reductive set $\mathrm{R}$ and $R=\{B\}$. 
Perform the next loop with two attributes, we have $\gamma_{\{A, B\}}^{\prime}(Q)=3.4 / 6, \quad \gamma_{\{B, C\}}^{\prime}(Q)=3.2 / 6$. So

$S I G_{B}(A)=\gamma_{\{B, A\}}^{\prime}(D)-\gamma_{B}^{\prime}(D)=3.4 / 6-2.4 / 6=1 / 6$

$S I G_{B}(C)=\gamma_{\{B, C\}}^{\prime}(D)-\gamma_{B}^{\prime}(D)=3.2 / 6-2.4 / 6=0.8 / 6$

Attribute $A$ is selected to add to collective set and we have $R=\{A, B\}$. Finally, included in the attributes $\mathrm{C}$ and caculated $\gamma_{\{A, B, C\}}^{\prime}(Q)=3.4 / 6$. This result does not change the dependency function, it means that $\gamma_{\{A, B, C\}}^{\prime}(Q)=\gamma_{\{A, B\}}^{\prime}(Q)$. Thus, algorithm stopped and $R=\{A, B\}$ is a minimal collection of decision table $D S$, it means that $R=\{a, b\}$ is a minimal reduction set of decision table. With this data set, performing reduction approach of traditional rough set using heuristic algorithm obtained results $R=\{A, B, C\}$.

Algorithm 1 shows immediately minimal reduction, the accuracy of classification stability than the rough set approach on the same data set, preserving the positive region, this issue published by the authors Jensen and Q. Shen [7] do not sastify, belong to NP-hard problem. The computational complexity large. Computational complexity layered fuzzy equivalent of the attributes is exponential $O\left(|U| \times c^{|A|}\right)$,

which $|U|$ is the cardinality of universe, $|A|$ is number of attributes, $\mathrm{c}$ is the number of linguistic terms describing each attribute. Therefore, Algorithm 1 only academic, not feasible to deploy realistic.

\section{ATTRIBUTES REDUCTION BASED ON THE DISCERNIBLITY MATRIX}

In this section, we introduce the algorithm finds all reductions of fuzzy decision table based on discernibility matrix. Suppose the fuzzy decision table $D S=(U, C \cup D)$ which $U=\left\{x_{1}, \ldots, x_{n}\right\}$ is a finite set of objects, $C=\left\{c_{1}, \ldots, c_{m}\right\}$ is a set of conditional attributes, $D$ is the set of decision attributes. $R_{k}$ is a fuzzy similarity relation which define on elements of condition attributes $c_{k} \in C, k=1 . . m$, the fuzzy relational matrix of conditional attributes $c_{k}$ is $M\left(R_{k}\right)=R_{k}\left(x_{i}, x_{j}\right)=\left[\mathrm{c}_{\mathrm{ij}}^{k}\right]_{n x n}$. Denote that $R$ is the family of fuzzy similarity of relations, the fuzzy decision table is rewritten in the form of fuzzy similar relationship $D S=(U, R \cup D)$, denote $\mathrm{M}(\mathrm{R})=\bigcap\left\{\mathrm{M}\left(R_{k}\right): R_{k} \in R\right\}=\underset{k=1 . . m}{\min }\left\{\mathrm{c}_{\mathrm{ij}}^{k}\right\}, \mathrm{M}(R)$ is also a fuzzy relational matrix. $R_{k}$ is dispensable relative to $D$ in $R$ if $\operatorname{Pos}_{\mathrm{M}(\mathbf{R})} D=\operatorname{Pos}_{(\mathrm{M}(\mathbf{R}-\{R\})} D$, otherwise $R_{k}$ is indispensable relative to $D . P \subseteq R$ is a reduct of $R$ relative to $D$ if $\operatorname{Pos}_{\mathrm{M}(\mathbf{R})} D=\operatorname{Pos}_{\mathrm{M}(\mathbf{P})} D$. The collection of all the indispensable elements relative $D$ in $R$ is called the core of $R$, denoted as Core ${ }_{D}(R)$.
Denoted $R_{*} A$ is $R$-lower approximation of the $A$ where $A$ is a fuzzy set on $U$ and $R$ is really a fuzzy similarity relation. Lower approximation of all objects $x \in U$ is caculated [3], [10]:

$$
R_{*} A(x)=\inf _{y \in U} \max \{1-R(x, y), A(y)\}
$$

In fuzzy sets, a fuzzy point $x_{\lambda}$ is defined as

$$
x_{\lambda}(z)=\left\{\begin{array}{ll}
\lambda & z=x \\
0 & z \neq x
\end{array}, z \in U\right.
$$

Fuzzy set is union of fuzzy points.We define equivalence classes for all fuzzy $x_{\lambda}$ as the basic granule, as follows:

$$
\begin{aligned}
x_{\lambda}(z)=\left\{\begin{array}{rl}
\lambda & 1-R(x, z) \geq \lambda \\
0 & 1-R(x, z)<\lambda
\end{array}\right. \\
\quad z \in U, x \in U, \lambda \in(0,1]
\end{aligned}
$$

$R_{*} A$ is expressed through the equivalent class

$$
R_{*} A=\bigcup\left\{\left(x_{\lambda}\right)_{R}:\left(x_{\lambda}\right)_{R} \subseteq A, \lambda \notin[0,1]\right\}
$$

Let $\lambda=M(R)_{*}\left([x]_{D}\right)(x)$ denote $M(R)$ - lower approximation of $[x]_{D}, x \in U$. According to the nature of the positive region, $P \subset R$ is a reduct of $R$ if $\left(x_{\lambda}\right)_{M(\mathbf{P})} \subseteq[\mathrm{x}]_{D}$ where $\lambda=M(R)_{*}\left([x]_{D}\right)(x)$ [1], [2], [10].

Discernibility matrix $M_{D}(U, R)$ of $(U, R \cup D)$ is a $n \times n$ matrix $\left(c_{i j}\right)$. Like this:

1) if $\lambda_{j}<\lambda_{i}$ then $c_{i j}=\left\{R_{k}: 1-R_{k}\left(x_{i,} x_{j}\right) \geq \lambda_{i}\right\}$, where $\lambda_{i}=M(R)_{*}\left(\left[x_{i}\right]_{D}\right)\left(x_{i}\right), \lambda_{j}=M(R)_{*}\left(\left[x_{i}\right]_{D}\right)\left(x_{j}\right)$

2) $c_{i j}=\phi$, otherwise

A discernibility function $f_{D}(U, R)$ for $(U, R \cup D)$ is a boolean function of $m$ Boolean variable $\bar{R}_{1}, \ldots, \bar{R}_{m}$ corresponding to the fuzzy attributes $R_{1}, \ldots, R_{m}$, respectively is defined as follows [2, 10]:

$$
f_{D}(U, R)\left(\overline{R_{1}}, \ldots, \overline{R_{m}}\right)=\wedge\left\{\vee\left(c_{i j}\right): c_{i j} \neq \phi\right\}
$$

Algorithm 2. The algorithm finds all reductions for fuzzy decision system as follows:

Suppose $U=\left\{x_{1}, \ldots, x_{n}\right\}, U / D=\left\{D_{1}, \ldots, D_{s}\right\}$

1. Calculate $\mathrm{M}(\mathrm{R})$

2. Calculate $\mathrm{M}(R)_{*}\left(D_{k}\right)$ with all $D_{k} \in U / D$

3. Calculate $c_{i j}:$ If $\lambda_{j}<\lambda_{i}$ then $c_{i j}=\left\{R_{k}: 1-R_{k}\left(x_{i}, x_{j}\right) \geq \lambda_{i}\right\}$, otherwise $c_{i j}=\phi$ 
4. Calculate $\operatorname{Core}_{D}(R)$ of single element $c_{i j}$

5. Delete those $c_{i j}=\phi$ or $c_{i j} \cap$ Core $\neq \phi$

6. Define $f_{D}(U, R)=\wedge\left\{\vee\left(c_{i j}\right)\right.$ with $c_{i j}$ collect from step 5

7. Calculate $g_{D}(U, R)=\left(\wedge R_{1}\right) \vee \ldots \vee\left(\wedge R_{1}\right)$ with

$f_{D}(U, R)=\left\{\vee\left(c_{i j}\right)\right.$

8. All reductions: $\operatorname{Core}_{D}(R) \wedge\left\{\vee\left(c_{i j}\right)\right.$

Applies Algorithm 2 for table 2, with all the attributes defined by fuzzy similarity relations as follows:

$R_{k}\left(x_{i}, x_{j}\right)= \begin{cases}\left.\min \left\{c_{k}\left(x_{i}\right), c_{k}\left(x_{j}\right)\right)\right\}, & c_{k}\left(x_{i}\right) \neq c_{k}\left(x_{j}\right) \\ 1, & c_{k}\left(x_{i}\right)=c_{k}\left(x_{j}\right)\end{cases}$

The results were fuzzy similarity matrix $M\left(R_{1}\right)\left(x_{i}, x_{j}\right)$, $M\left(R_{2}\right)\left(x_{i}, x_{j}\right), M\left(R_{3}\right)\left(x_{i}, x_{j}\right), M\left(R_{4}\right)\left(x_{i}, x_{j}\right)$,

$M\left(R_{5}\right)\left(x_{i}, x_{j}\right), M\left(R_{6}\right)\left(x_{i}, x_{j}\right)$.

Caculate $\mathrm{M}(R)\left(x_{i}, x_{j}\right)=\left(\begin{array}{cccccc}1 & 0 & 0 & 0 & 0 & 0 \\ 0 & 1 & 0 & 0 & 0 & 0 \\ 0 & 0 & 1 & 0 & 0 & 0 \\ 0 & 0 & 0 & 1 & 0.4 & 0 \\ 0 & 0 & 0 & 0.4 & 1 & 0 \\ 0 & 0 & 0 & 0 & 0 & 1\end{array}\right)$

Partition of decision attribute $D=\{d\}$ is $D_{1}=\left\{x_{1}, x_{3}, x_{6}\right\}$, $D_{2}=\left\{x_{2}, x_{4}, x_{5}\right\}$

$$
\begin{aligned}
M(R)_{*}\left(D_{1}(x)\right) & =\left(\begin{array}{ll}
1, & x=x_{1} \\
0, & x=x_{2} \\
1, & x=x_{3} \\
0, & x=x_{4} \\
0, & x=x_{5} \\
1, & x=x_{6}
\end{array}\right. \\
M(R)_{*}\left(D_{2}(x)\right) & =\left(\begin{array}{ll}
0, & x=x_{1} \\
1, & x=x_{2} \\
0, & x=x_{3} \\
1, & x=x_{4} \\
1, & x=x_{5} \\
0, & x=x_{6}
\end{array}\right.
\end{aligned}
$$

Calculated $c_{i j}$ by Algorithm 2 through $R_{k}\left(x_{i}, x_{j}\right), \lambda_{i}, \lambda_{j},(\mathrm{i}, \mathrm{j}$, k) $=1 . .6$, a discernibility matrix obtained:

$$
M_{D}(U, R)=c_{i j}=\left(\begin{array}{cccccc}
\phi & \{3,6\} & \phi & \{1,5,6\} & \{1,5,6\} & \phi \\
\{3,6\} & \phi & \{3\} & \phi & \phi & \{1,5\} \\
\phi & \{3\} & \phi & \{1,5\} & \{1,5\} & \phi \\
\{1,5,6\} & \phi & \{1,5\} & \phi & \phi & \{3\} \\
\{1,5,6\} & \phi & \{1,5\} & \phi & \phi & \{3\} \\
\phi & \{1,5\} & \phi & \{3\} & \{3\} & \phi
\end{array}\right)
$$

Core $_{D}(R)=\{3\}, f_{D}(U, R)=(1 \vee 5), \quad R_{1}=\{1,3\}$, $R_{2}=\{3,5\}$. All reducts are $R_{1}=\{a, b\}, R_{2}=\{b, c\}$

Algorithm 2 finds all reductions of conditional attributes but not know the minimal subset, this issue was mentioned in the publication of the group Eric C.C Tsang, Chen Degang [10] but not accurate in the process of finding reduction, belong NP-hard problem. Implementation time is faster than Algorithm 1 in the same dataset. The computational complexity of indiscernibility relation $\mathrm{M}(\mathrm{R})$ and lower approximation $\left(\mathrm{M}(\mathrm{R})_{*}(D)_{k}, \forall D_{k} \in U / D\right) \quad$ is $O\left((|A|+|D|) \times|U|^{2}\right)$, where $|U|$ denotes the size of universe; $|A|$ denotes the number of attributes; $|D|$ denotes the number of decision classes. Algorithm 2 needs more memory space to store to calculate similarity relations and lower approximation $O\left(|U|^{2}\right)$ and $O\left(|U|^{2} \times|D|\right)$. Thus, Algorithm 2 is feasible in practical application when finding all reductions of fuzzy decision table.

\section{CONCLUSIONS}

Model of fuzzy rough set by D. Dubois and colleagues suggested that the combination of rough set theory and fuzzy set theory. Rough set theory preserves vagueness in data while fuzzy set theory preserves semantics (fuzziness) in data. Therefore, fuzzy rough set tools is considered more effective in rough set tools of attributes reduction and extracted law on information systems which have continued or semantic values attribute value domain. In this paper, based on attributes reduction method using the dependency function between attributes in rough set theory, we improved attribute reduction methods in decision table using fuzzy the degree of dependency between attributes, discernibility matrix approach fuzzy rough set and assess the advantages and disadvantages of each method, overcome the limitations and mistakes of the authors studied the issue. Two illustrative examples on the same dataset demonstrate that fuzzy rough set approach is more effective than traditional rough set theory. Our oriented research is constructed an effective attribute reduction methods in fuzzy decision table according to fuzzy rough set approach and experimental methods with the actual problem

\section{REFERENCES}

[1] Nguyen Long Giang, Rough Set Based Data Mining Methods, Doctor of Thesis, Institute of Information Technology, 2012.

[2] D. Dubois and H. Prade, Putting rough sets and fuzzy sets together, Intelligent Decision Support, Kluwer Academic Publishers, Dordrecht, 1992.

[3] Dubois, D. and Prade, H., Rough fuzzy sets and fuzzy rough sets, International Journal of General Systems, 17, pp. 191-209, 1990.

[4] L. A. Zadeh, Fuzzy sets, Information and Control, 8:338 $353,1965$. 
[5] Pawlak Z., Rough sets, International Journal of Computer and Information Sciences, 11(5): 341-356, 1982.

[6] Pawlak Z., Rough sets: Theoretical Aspects of Reasoning About Data, Kluwer Aca-demic Publishers, 1991.

[7] R. Jensen and Q. Shen., Fuzzy-Rough Sets for Descriptive Dimensionality Reduction, Proceedings of the 11th International Conference on Fuzzy Systems, pp. $29-34,2002$.
[8] Yao, Y.Y., On combining rough and fuzzy sets, Proceedings of the CSC'95 Workshop on Rough Sets and Database Mining, Lin, T.Y. (Ed.), San Jose State University, 9 pages, 1995 .

[9] Y.Y. Yao, A Comparative Study of Fuzzy Sets and Rough Sets, Vol.109, 21-47, Information Sciences. 1998.

[10] Eric C. C. Tsang, Degang Chen, Daniel S. Yeung, XiZhao Wang, and John W. T. Lee, Attributes Reduction Using Fuzzy Rough Sets, IEEE Transactions On Fuzzy Systems, Vol. 16, No. 5, Octerber 2008. 